\title{
Infrared Spectroscopic Characterization of CIT-6 and a Family of * BEA Zeolites
}

\author{
Sean R. Tomlinson, ${ }^{1}$ Tyler McGown, ${ }^{2}$ John R. Schlup, ${ }^{1}$ and Jennifer L. Anthony ${ }^{1}$ \\ ${ }^{1}$ Department of Chemical Engineering, Kansas State University, 1005 Durland Hall, Manhattan, KS 66506-5102, USA \\ ${ }^{2}$ Burns \& McDonnell, 9400 Ward Parkway, Kansas City, MO 64114, USA \\ Correspondence should be addressed to Jennifer L. Anthony; anthonyj@ksu.edu
}

Received 26 June 2013; Accepted 9 September 2013

Academic Editor: Akihide Wada

Copyright (c) 2013 Sean R. Tomlinson et al. This is an open access article distributed under the Creative Commons Attribution License, which permits unrestricted use, distribution, and reproduction in any medium, provided the original work is properly cited.

\begin{abstract}
Infrared spectroscopy is known to be a useful tool for identifying local structure changes in zeolites. Infrared spectroscopy is often employed to complement X-ray diffraction data. Local structure changes in zeolite CIT- 6 and its zeolite beta $\left({ }^{*} \mathrm{BEA}\right)$ analogs caused by calcination, altering framework composition, and ion exchange have been identified with mid- and far-infrared spectroscopy. Differences in the local structures of the samples were observed in mid- and far-infrared spectra, including changes in the intratetrahedral asymmetric stretch, the double-ring mode, and the intratetrahedral bending mode regions. The infrared spectra indicate that calcination or acetic acid extraction changed the structure of CIT- 6 to that of zeolite beta $\left({ }^{*} \mathrm{BEA}\right)$. Zinc ion exchange or the substitution of aluminum into the framework structure of acetic acid extracted samples retained the CIT- 6 structure.
\end{abstract}

\section{Introduction}

Microporous molecular sieves are employed in catalytic, ion exchange and adsorption/separation processes and are, therefore, of scientific and commercial interest [1]. While "zeolite" specifically refers to aluminosilicate microporous molecular sieves, the term is often applied to compounds with similar crystalline structures. Zeolites are remarkable because of their uniform pores (which are less than $2 \mathrm{~nm}$ ), their channel configuration, and their void space organization.

Because of zeolites' vast application potential, the characterization of chemical composition, structure, and bonding are important, leading to the use of various analytical techniques to obtain such data. Chemical composition of zeolites can be obtained with atomic absorption spectroscopy (AAS), atomic emission spectrometry (ICP-AES), and Xray fluorescence spectrometry (XRF) [2]. Both ${ }^{29} \mathrm{Si}$ and ${ }^{27} \mathrm{Al}$ nuclear magnetic resonance (NMR) spectroscopies have proven to be valuable tools in understanding local environments surrounding atoms in the lattice. When NMR data are coupled with density functional theory (DFT) calculations, valuable insights into the crystalline structure of zeolites result [3]. X-ray diffraction (XRD) is also widely used to determine crystalline structure of zeolitic materials, and these data permit identification of samples by comparison with $\mathrm{XRD}$ results of known samples [4-6].

Vibrational spectroscopy, including both Raman and infrared (IR), is often employed to investigate the chemistry (with probe molecules) and short-range order in zeolites [715]. In the 1970s, Flanigen et al. proposed zeolite functional group assignments for the absorption bands between 1250 and $300 \mathrm{~cm}^{-1}[14,15]$. Assignments divided spectra into regions classified with respect to intra- or intertetrahedral vibrations. Intratetrahedral vibrations are stretches associated with movements within the $\mathrm{TO}_{4}$ tetrahedra that comprise the zeolite's structure. These movements can be $\mathrm{O}-\mathrm{T}-\mathrm{O}$ bending modes or symmetric or asymmetric stretches of $\mathrm{O}-$ $\mathrm{T}-\mathrm{O}$ bonds. Intertetrahedral (between adjacent tetrahedra) vibrations include pore-opening (also known as breathing) modes. These guidelines have become the primary tool for absorption assignments in the infrared spectra of zeolites $[13,16]$. Far-infrared spectroscopy (FIR) (wavenumbers less than $400 \mathrm{~cm}^{-1}$ ) has been used to probe the local cation environment [17], provide structural information such as 
pore-breathing and intratetrahedral bending modes on the zeolite network [14], and examine the local environment of zeolites through cation probes [17-20].

The present study examines the infrared spectra of the zincosilicate molecular sieve CIT-6. CIT-6 was first synthesized and characterized in 1999 [21] and has since been examined with respect to chemical properties [6], mechanism of crystallization [22], and ability to catalyze propane dehydrogenation [4]. CIT-6 is of interest in this study as it can be transformed from the original zincosilicate to a pure silicate without changing the XRD spectra of the sample [21]. This pure silicate can subsequently be modified, without visibly altering the XRD spectra to an aluminosilicate through the insertion of aluminum into T-atom locations formerly occupied by zinc [21]. However, no investigation has been done as to how these chemical changes affect shortrange structure, that is, structure changes that may occur and affect zeolite performance, but are not visible using XRD.

CIT-6 has the framework structure of zeolite beta (International Zeolite Association designation * BEA), which is particularly appealing for study because it is an ingrowth of two polymorphs $[21,23]$. Zeolite * BEA is also useful in industrial applications such as gas separation and reforming of industrial naphtha $[24,25]$. Zeolite ${ }^{*} \mathrm{BEA}$ has been analyzed for long-range order in development of its polymorphs [26], short-range order with ${ }^{29} \mathrm{Si}$ NMR chemical shifts to identify structure [3], effects of partial replacement of silicon with tin or titanium atoms [27], and development of connectivitydefect-free zeolite ${ }^{*}$ BEA [28]. Structural [7, 29, 30] and chemical properties $[31,32]$ of pure silica zeolite ${ }^{*}$ BEA have been investigated using infrared spectroscopy. However, no reported attempts have been made to analyze structure or chemical properties of CIT-6 and modified versions using infrared spectroscopy. Of particular interest is whether IR, especially FIR, can be employed to view local changes to the structure that are otherwise difficult to see using only XRD.

In this work, vibrational spectra of CIT- 6 and its derivatives are reported over the spectral range from $1500 \mathrm{~cm}^{-1}$ to $200 \mathrm{~cm}^{-1}$. Seven samples are included in this report: (1) as synthesized, prior to any treatments (As-made CIT-6); (2) assynthesized after calcination (Calcined); (3) low-temperature $\left(60^{\circ} \mathrm{C}\right)$ acetic acid structural-zinc extracted (Low- $T$ Si $\left.{ }^{*} \mathrm{BEA}\right)$; (4) high-temperature $\left(135^{\circ} \mathrm{C}\right)$ acetic acid structural-zinc extracted (High-T Si $\left.{ }^{*} \mathrm{BEA}\right)$; (5) low-temperature acetic acid with aluminum inserted into the T-atoms structural locations from which zinc had been extracted (Aluminum); (6) as synthesized that has been ion-exchanged to substitute zinc for the original lithium cation (Zn CIT-6); and (7) pure silica *BEA (Pure Si *BEA) $[4,6,28]$.

\section{Experimental Section}

CIT-6 was synthesized following the procedure described by Takewaki et al. [21] Reactants were mixed at a molar composition of 0.05 moles $\mathrm{LiOH}: 0.03$ moles $\mathrm{Zn}(\mathrm{OAc})_{2}$ (zinc acetate):0.65 moles TEAOH (tetraethylammonium hydroxide) : 1 mole $\mathrm{SiO}_{2}: 30$ moles $\mathrm{H}_{2} \mathrm{O}$ and heated for four days at $150^{\circ} \mathrm{C}$ in a Teflon-lined autoclave. The product was recovered via filtration, washed with water, and dried at $100^{\circ} \mathrm{C}$ overnight in air. This "As-made CIT-6" is a zincosilicate zeolite with lithium cations to balance the framework charge. It also contains residual $\mathrm{TEAOH}$ in pores that serve as the structure-directing agent (SDA). Postsynthesis structure analysis was performed with either a Bruker AXS D8 Advance X-ray diffractometer or a Rigaku MiniFlex II X-ray diffractometer, using copper $\mathrm{K} \alpha$ radiation.

The As-made CIT- 6 was then exposed to five treatments to modify framework composition while maintaining the ${ }^{*}$ BEA structure visible with XRD [6]. Figure 1 shows these treatments and the resulting samples. The "Calcined" sample was obtained by heating As-made CIT- 6 at $550^{\circ} \mathrm{C}$ for ten hours to remove remaining SDA [4]. The "Zn CIT-6" was prepared by ion-exchanging lithium cations in the As-made CIT-6 with zinc cations through exposure to a 1 molar $\mathrm{Zn}\left(\mathrm{NO}_{3}\right)_{2}$ solution for ten hours at $80^{\circ} \mathrm{C}$ [4]. In both the "High-T Si * BEA" and "Low-T Si ${ }^{*} B E A$," intratetrahedral zinc atoms were extracted from the As-made CIT-6 zincosilicate framework with acetic acid, leaving behind a pure silica zeolite with the ${ }^{*} \mathrm{BEA}$ framework [6]. The High-T Si ${ }^{*} \mathrm{BEA}$ resulted from exposing As-made CIT- 6 to acetic acid at $135^{\circ} \mathrm{C}$ for two days, where the higher temperature is expected to anneal extracted zinc sites [6]. The Low- $T$ Si ${ }^{*} \mathrm{BEA}$ was made by exposing As-made CIT- 6 to acetic acid at $60^{\circ} \mathrm{C}$ for three days, resulting in exposed silanol groups at the sites of extracted zinc $[6,21]$. Mixing the Low-T Si ${ }^{*}$ BEA with aluminum nitrate nonahydrate at a $1: 2.50$ weight ratio of zeolite to $\mathrm{Al}\left(\mathrm{NO}_{3}\right)_{3} \cdot 9 \mathrm{H}_{2} \mathrm{O}$ at $80^{\circ} \mathrm{C}$ for one day resulted in the insertion of an aluminum atom into the tetrahedral framework [6], thus creating the aluminosilicate form of zeolite *BEA, referred to here as "Aluminum."

The "Pure Si *BEA," a pure silica zeolite *BEA, was synthesized following the procedure described by Camblor et al. [28]. This is the only sample studied in this work that did not originate from postsynthesis treatments of As-made CIT-6. Pure $\mathrm{Si}{ }^{*} \mathrm{BEA}$ was synthesized using hydrofluoric acid, resulting in formation of zeolite ${ }^{*} \mathrm{BEA}$ with enhanced crystallinity and no connectivity defects [28]. This is an excellent model for comparison of ${ }^{*}$ BEA structure with other samples in this study [28].

Infrared spectroscopic data were obtained with a Mattson Instruments Cygnus 100 FTIR spectrometer. All data were obtained via diffuse reflection utilizing a Spectra Tech "COLLECTOR" diffuse reflection accessory. Collected data were obtained in Kubelka-Munk units, the preferred units of diffuse reflectance IR, and then normalized to account for variations in spectral intensity arising from the dilution occurring during sample preparation $[33,34]$.

In the mid-infrared (MIR) region, a high-temperature ceramic source and a germanium-coated potassium bromide $(\mathrm{KBr})$ beam splitter were employed. The detector was a deuterated triglyceride sulfate (DTGS) detector with a $\mathrm{KBr}$ window. The mirror velocity was $0.6 \mathrm{~cm} / \mathrm{sec}$, and the resolution was set at two $\mathrm{cm}^{-1}$. The iris setting was $50 \%$. One thousand twenty-four scans were coadded for each spectrum.

In the far-infrared (FIR) region, a high-temperature ceramic source was employed. The beam splitter was a threemicron thick Mylar film, and the detector was a DTGS detector with a polyethylene window. The mirror velocity was 


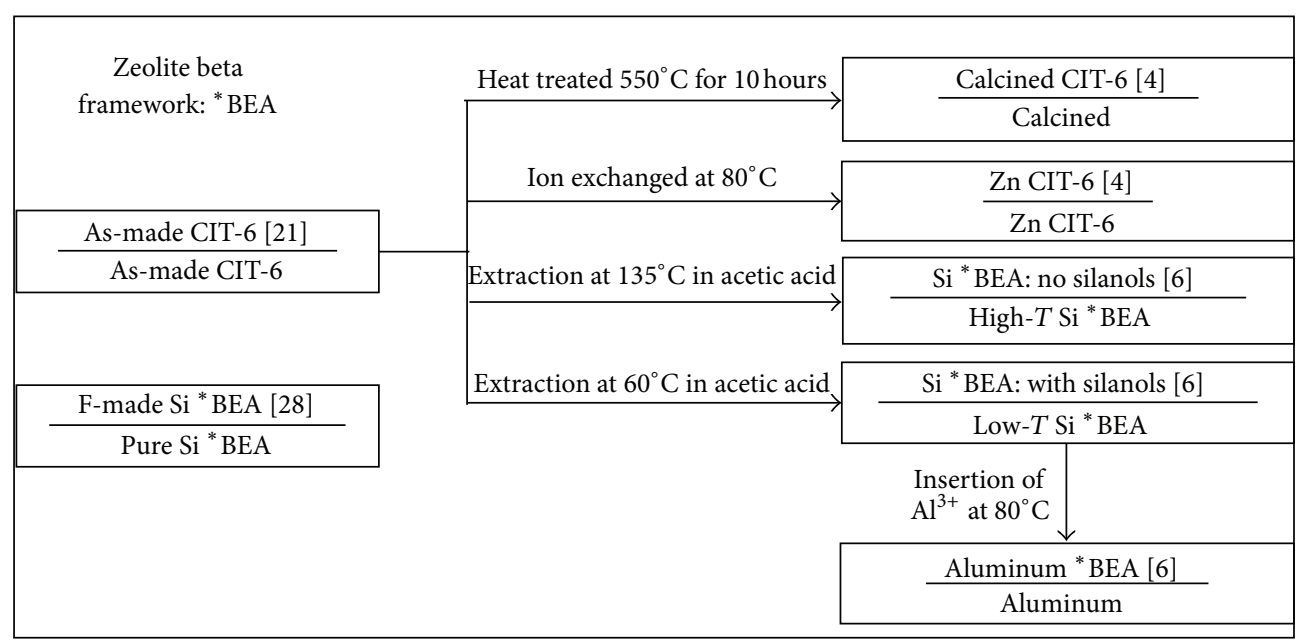

FIGURE 1: Flow chart of synthesis procedures from Andy and Davis [4], Takewaki et al. [6, 21], and Camblor et al. [28].

$0.3 \mathrm{~cm} / \mathrm{sec}$, and the resolution was two $\mathrm{cm}^{-1}$. The iris was $50 \%$ open. Each spectrum was the result of coadding 1,024 scans.

Due to requirements of the diffuse reflection technique, all samples were mixed with cesium iodide powder at an approximate ratio of 1:5 (zeolite: CsI). A mortar and pestle were used to reduce the particle size of the solids and to mix cesium iodide powder and sample. CsI powder was used for the background spectra.

\section{Results and Discussion}

As reported in the literature, XRD patterns of the samples did not change significantly upon treatment [4]. Figure 2 shows similarities between the As-made CIT-6, Calcined, and Aluminum samples; the XRD pattern of the defect-free Pure $\mathrm{Si}^{*} \mathrm{BEA}$ is included for comparison [4]. All patterns exhibit strong similarities, with the only difference between spectra being the appearance of peaks at $13.4^{\circ}$ and $14.4^{\circ}$ $2 \theta$ in the Calcined sample versus the Aluminum and Asmade samples. These differences are consistent with results reported in the literature and are similar to peaks seen in the XRD of the Pure $\mathrm{Si}^{*} \mathrm{BEA}$ sample [21].

The mid-infrared peak assignments from 1500 to 700 $\mathrm{cm}^{-1}$ are given in Table 1 , and MIR spectra for all samples can be seen in Figure 3. The As-made CIT-6 and Pure Si * BEA sample spectra match spectra reported in the literature $[22,35]$.

In Figure 3 all the samples (except Pure $\mathrm{Si}^{*} \mathrm{BEA}$ ) show absorptions in the aliphatic $\mathrm{C}-\mathrm{H}$ bending mode region between 1500 and $1350 \mathrm{~cm}^{-1}$, characteristic of either leftover SDA or its decomposition products [4, 36, 37]. The presence of residual SDA is consistent with a previous report that $24 \%$ of SDA remains after acetic acid extraction [4]. The Aluminum sample is unique, having a more complex spectrum in the region from 1500 to $1250 \mathrm{~cm}^{-1}$. The additional, medium intensity, broad peaks in the Aluminum sample between 1500 and $1250 \mathrm{~cm}^{-1}$ are due to nitrate anions from the aluminum insertion process $[36,37]$. Additionally,

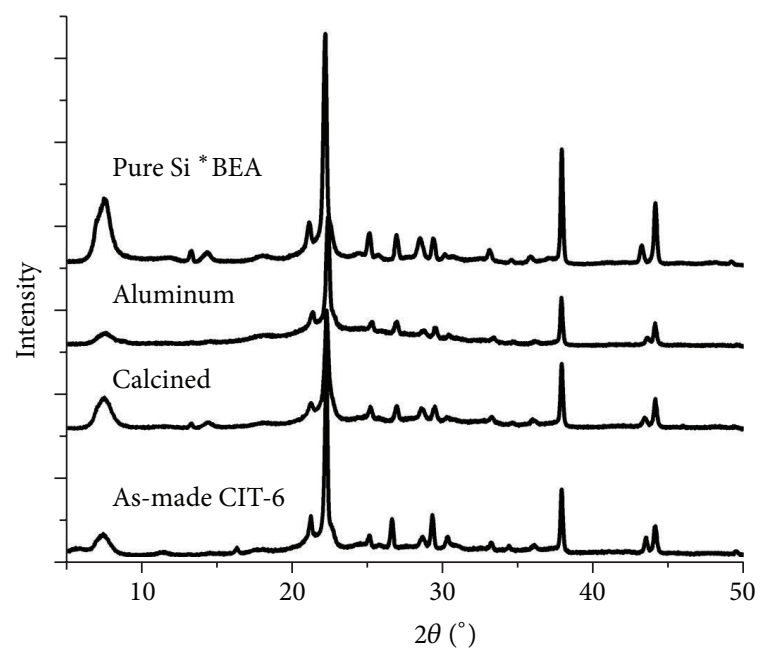

FIgure 2: XRD results for Pure Si * BEA, Aluminum, Calcined, and As-made CIT-6 samples.

the High-T and Low- $T$ Si ${ }^{*}$ BEA and Aluminum samples have small peaks at approximately $950 \mathrm{~cm}^{-1}$, indicative of residual acetic acid [37].

Differences in the spectra in Figure 3 show changes in the intratetrahedral asymmetric O-T-O region, between 1230 and $990 \mathrm{~cm}^{-1}$, indicating a change in the intratetrahedral environment. The As-made CIT-6 structure is retained during zinc ion exchange (sample $\mathrm{Zn}$ CIT-6), as shown by the similarity in the peaks at 1165 and $998 \mathrm{~cm}^{-1}$. But these peaks are absent in the Calcined, High- $T$, and Low- $T$ samples. Either calcination or acetic acid extraction results in spectra more similar to the Pure $\mathrm{Si}^{*} \mathrm{BEA}$ sample, particularly with regard to disappearance of the peak at $1165 \mathrm{~cm}^{-1}$. However, the Aluminum sample, which inserted aluminum into vacant zinc locations (caused by acetic acid extraction), apparently has a small peak at $1165 \mathrm{~cm}^{-1}$, indicating a return to an intratetrahedral environment similar to the original CIT-6. 


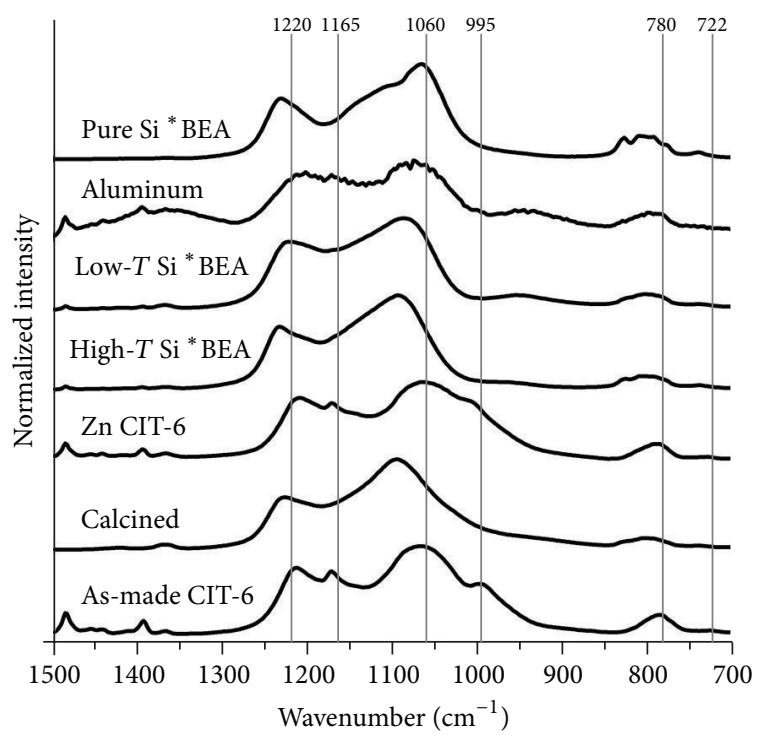

FIgURE 3: Mid-infrared spectra of samples with sample labels and peaks of interest labeled.

All spectra show a broad region between two primary peaks at $\sim 1220$ and $\sim 1070 \mathrm{~cm}^{-1}$, but only the Pure $\mathrm{Si}{ }^{*}$ BEA sample shows an additional broad peak at $1103 \mathrm{~cm}^{-1}$. The structural changes shown in Figure 3 for the samples are not seen in the XRD of the samples (Figure 2). The differences between the spectra shown in Figure 3 indicate the usefulness of IR to show short-range differences in zeolite structure as opposed to the long-range structure identification ability of XRD.

All samples have a peak at approximately $800 \mathrm{~cm}^{-1}$ and a smaller peak or shoulder at approximately $735 \mathrm{~cm}^{-1}$, identified by Flanigen as symmetric intertetrahedral $\mathrm{O}-\mathrm{T}-\mathrm{O}$ stretches $[14,15]$. This similarity indicates little change in intertetrahedral $\mathrm{O}-\mathrm{T}-\mathrm{O}$ bonding of the samples, in spite of the intratetrahedral changes discussed above.

Far-infrared peak assignments are given in Table 2, and the FIR spectra are provided in Figure 4.

The Pure $\mathrm{Si}$ * BEA sample has an FIR spectrum consistent with previous reports in the literature [35]. Far-IR spectra for CIT- 6 and its derivatives have not been previously reported. Figure 4 shows a change in the double-ring mode region (from 650 to $500 \mathrm{~cm}^{-1}$ ) for the Calcined and High-T and Low- $T \mathrm{Si}^{*} \mathrm{BEA}$ samples with the addition of the extra peak at $640 \mathrm{~cm}^{-1}$, compared to the original As-made CIT-6 [14, 15]. The region from 500 to $420 \mathrm{~cm}^{-1}$ also shows changes in the intratetrahedral $\mathrm{T}-\mathrm{O}$ bending region for High- $T$ and Low$T \mathrm{Si}^{*} \mathrm{BEA}$ and Calcined samples with an additional peak or shoulder at approximately $473 \mathrm{~cm}^{-1}$, compared to As-made CIT-6 [14, 15]. The $473 \mathrm{~cm}^{-1}$ peak is clearly resolved in the Pure $\mathrm{Si}^{*}$ BEA spectrum. Similar to changes in the MIR shown in Figure 3, calcination and acetic acid extraction result in a change in the FIR and together indicate a change in the structure of the zeolite.

As in the MIR shown in Figure 3, the similarity of Aluminum's FIR structure to that of As-made CIT-6 is interesting because the Aluminum sample is prepared from the Low- $T \mathrm{Si}$

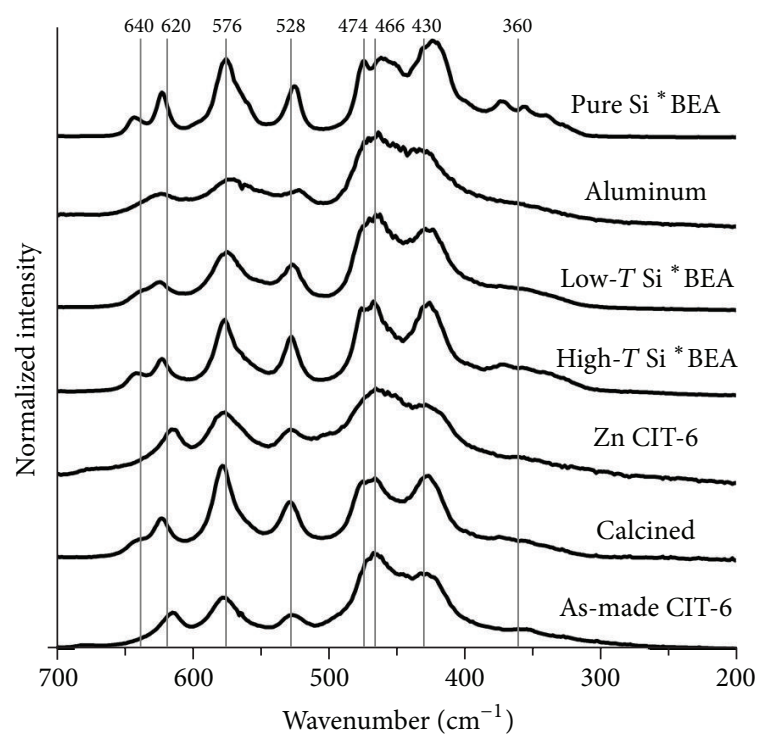

FIGURE 4: Far-infrared spectra of samples with sample labels and peaks of interest labeled.

${ }^{*} \mathrm{BEA}$. As the Low- $T \mathrm{Si}{ }^{*} \mathrm{BEA}$ sample shows changes from the original As-made CIT-6, the return to the original structure is an interesting outcome of the aluminum insertion process. This change shows that inserting aluminum atoms into Tatom locations once occupied by zinc results in reversion of the framework structure from Low- $T \mathrm{Si}^{*} \mathrm{BEA}$ to the original As-made CIT-6 sample. This structural reversibility is of note because changes are visible with IR, even though zinc T-atom locations are only $3 \%$ of total T-atom sites available [4].

The region from 420 to $300 \mathrm{~cm}^{-1}$ shows the pore-opening modes of the zeolites $[14,15]$. The Pure $\mathrm{Si}^{*} \mathrm{BEA}$ sample has three clear peaks, and the High- $T \mathrm{Si}^{*}$ BEA exhibits three weak peaks at the same wavenumbers. Other samples show little difference from As-made CIT-6. Similarities of As-made CIT6 and its derivatives in this region suggest that these samples lack long-range order at the level of pore-breathing modes observed with the Pure $\mathrm{Si}^{*} \mathrm{BEA}$ sample. Lack of change in the pore-opening region corroborates what is seen in XRD results where samples maintain similar patterns even after treatment [21].

The synthesis method for Pure $\mathrm{Si}$ * BEA results in defectfree material, and a lack of defects contributes to resolution narrowing of absorption bands in this spectrum [28]. A narrowing of line widths for CIT-6 upon calcination is expected. Both Aluminum and Zn CIT-6 samples exhibit peak broadening in the FIR. The Aluminum sample's broader absorbances in the FIR are indicative of distortions of tetrahedral bond angles due to the extraction and insertion process. Despite the fact that only about $3 \%$ of the tetrahedral sites are impacted by replacing zinc with aluminum, the process of extraction and then reinsertion of the T-atoms creates a disruption in the long-range structure as evidenced by the broadening of both the mid- (Figure 3 ) and far-IR (Figure 4) spectra. The Zn CIT-6 samples show that limited ion exchange has the distorted local order of the samples even without disordering the overall structure. 
TABLE 1: Mid-IR peaks for CIT-6 and its derivatives. The notations are as follows: $\mathrm{w}$ is weak intensity, $\mathrm{m}$ is medium intensity, $\mathrm{s}$ is strong intensity, and sh is shoulder.

\begin{tabular}{|c|c|c|c|c|c|c|c|}
\hline As-made CIT-6 & Calcined & Zn CIT-6 & High- $T$ Si ${ }^{*} \mathrm{BEA}$ & Low- $T$ Si ${ }^{*}$ BEA & Aluminum & Pure $\mathrm{Si}^{*} \mathrm{BEA}$ & Assignment \\
\hline $1484 \mathrm{~m}$ & & $1484 \mathrm{~m}$ & $1484 \mathrm{w}$ & $1484 \mathrm{w}$ & & & \multirow{6}{*}{$\begin{array}{l}\delta \mathrm{C}-\mathrm{H} \text { and } \mathrm{NO}_{3}^{-} \text {(for Aluminum } \\
\text { sample only) }[36,37]\end{array}$} \\
\hline $1454 \mathrm{w}$ & & $1455 \mathrm{w}$ & & & $1459 \mathrm{~m}$ & & \\
\hline & & $1441 \mathrm{w}$ & & & $1438 \mathrm{~m}$ & & \\
\hline $1393 \mathrm{~m}$ & & $1394 \mathrm{w}$ & $1394 \mathrm{w}$ & & $1394 \mathrm{~m}$ & & \\
\hline \multirow[t]{2}{*}{$1367 \mathrm{w}$} & $1369 \mathrm{w}$ & $1366 \mathrm{w}$ & $1366 \mathrm{w}$ & $1367 \mathrm{w}$ & $1366 \mathrm{~m}$ & & \\
\hline & & & & & $1352 \mathrm{~m}$ & & \\
\hline $1207 \mathrm{~s}$ & $1220 \mathrm{~s}$ & $1203 \mathrm{~s}$ & $1216 \mathrm{~s}$ & $1226 \mathrm{~s}$ & $1200 \mathrm{~s}$ & $1226 \mathrm{~s}$ & \\
\hline \multirow[t]{2}{*}{$1165 \mathrm{~s}$} & & $1165 \mathrm{~s}$ & & & $1165 \mathrm{~m}$ & & \\
\hline & & & & & & $1103 \mathrm{sh}, \mathrm{s}$ & $v_{a}(\mathrm{O}-\mathrm{T}-\mathrm{O})[14,15]$ \\
\hline $1060 \mathrm{~s}$ & $1088 \mathrm{~s}$ & $1056 \mathrm{~s}$ & $1080 \mathrm{~s}$ & $1086 \mathrm{~s}$ & $1070 \mathrm{~s}$ & $1060 \mathrm{~s}$ & \\
\hline \multirow[t]{3}{*}{$991 \mathrm{~s}$} & & $998 \mathrm{~s}$ & & & & & \\
\hline & & & $961 \mathrm{sh}, \mathrm{m}$ & $952 \mathrm{~m}$ & $949 \mathrm{~m}$ & & Acetic acid [37] \\
\hline & $820 \mathrm{sh}, \mathrm{m}$ & & $816 \mathrm{~m}$ & $820 \mathrm{sh}, \mathrm{m}$ & $821 \mathrm{sh}, \mathrm{w}$ & $820 \mathrm{~m}$ & \\
\hline $777 \mathrm{~m}$ & $792 \mathrm{~m}$ & $788 \mathrm{~m}$ & $795 \mathrm{~m}$ & $795 \mathrm{~m}$ & $789 \mathrm{~m}$ & $795 \mathrm{~m}$ & $v_{s}(\mathrm{O}-\mathrm{T}-\mathrm{O})[14,15]$ \\
\hline $718 \mathrm{w}$ & $732 \mathrm{w}$ & $723 \mathrm{w}$ & $731 \mathrm{w}$ & $731 \mathrm{w}$ & $728 \mathrm{w}$ & $730 \mathrm{w}$ & \\
\hline
\end{tabular}

TABLE 2: Far-IR peaks for CIT-6 and its derivatives. The notations are as follows: $w$ is weak intensity, $\mathrm{m}$ is medium intensity, $\mathrm{s}$ is strong intensity, and sh is shoulder.

\begin{tabular}{|c|c|c|c|c|c|c|c|}
\hline As-made CIT-6 & Calcined & Zn CIT-6 & High- $T$ Si ${ }^{*} B E A$ & Low- $T$ Si ${ }^{*} \mathrm{BEA}$ & Aluminum & Pure $\mathrm{Si}^{*} \mathrm{BEA}$ & Assignment \\
\hline & $640 \mathrm{sh}, \mathrm{m}$ & & $640 \mathrm{w}$ & $638 \mathrm{sh}, \mathrm{w}$ & & $642 \mathrm{w}$ & \\
\hline $614 \mathrm{~m}$ & $622 \mathrm{~m}$ & $614 \mathrm{~m}$ & $622 \mathrm{~m}$ & $624 \mathrm{w}$ & $622 \mathrm{w}$ & $622 \mathrm{~m}$ & Double-ring modes $[14,15]$ \\
\hline $577 \mathrm{~m}$ & $577 \mathrm{~s}$ & $576 \mathrm{~s}$ & $576 \mathrm{~s}$ & $574 \mathrm{~m}$ & $569 \mathrm{~m}$ & $575 \mathrm{~s}$ & (1) \\
\hline \multirow[t]{2}{*}{$526 \mathrm{~m}$} & $528 \mathrm{~m}$ & $527 \mathrm{~m}$ & $527 \mathrm{w}$ & $526 \mathrm{~m}$ & $521 \mathrm{w}$ & $524 \mathrm{w}$ & \\
\hline & $474 \mathrm{~s}$ & $473 \mathrm{sh}, \mathrm{w}$ & $475 \mathrm{~s}$ & $473 \mathrm{sh}, \mathrm{s}$ & $472 \mathrm{sh}, \mathrm{s}$ & $474 \mathrm{~s}$ & \\
\hline $466 \mathrm{~s}$ & $465 \mathrm{~s}$ & $463 \mathrm{~s}$ & $466 \mathrm{~s}$ & $463 \mathrm{~s}$ & $463 \mathrm{~s}$ & $461 \mathrm{~s}$ & $\delta(\mathrm{T}-\mathrm{O})[14,15]$ \\
\hline $431 \mathrm{~s}$ & $427 \mathrm{~s}$ & $429 \mathrm{~s}$ & $426 \mathrm{~s}$ & $429 \mathrm{~s}$ & & 423 vs & \\
\hline \multirow{3}{*}{$360 \mathrm{w}$} & $374 \mathrm{w}$ & & 373 sh, w & $373 \mathrm{sh}, \mathrm{w}$ & & $373 \mathrm{w}$ & \\
\hline & $357 \mathrm{sh}, \mathrm{w}$ & $362 \mathrm{w}$ & $357 \mathrm{sh}, \mathrm{w}$ & & & $355 \mathrm{w}$ & Pore-opening modes $[14,15]$ \\
\hline & & & $342 \mathrm{sh}, \mathrm{w}$ & $347 \mathrm{sh}, \mathrm{w}$ & $350 \mathrm{sh}, \mathrm{m}$ & $340 \mathrm{w}$ & \\
\hline
\end{tabular}

Figure 4 demonstrates that the chemical or thermal treatments studied herein affect the structure of the sample by modifying both the intratetrahedral $\mathrm{T}-\mathrm{O}$ bending modes and the intertetrahedral double-ring modes. These changes can be seen in the FIR spectra of the sample. These changes in both the double-ring and intratetrahedral regions verify that chemical or thermal treatment converted As-made CIT-6 to the * BEA structure, while ion-exchanged Zn CIT- 6 does not exhibit a structural change [32].

\section{Conclusions}

The mid- and far-infrared spectra of CIT- 6 and related framework structures have been reported and band assignments have been made. Calcination or acetic acid extraction of CIT6 results in a structure different from the original As-made CIT-6 and very similar to Pure Si ${ }^{*} \mathrm{BEA}$ in both MIR and FIR. Pure Si ${ }^{*} \mathrm{BEA}$, Calcined, and Low-T and High-T Si
* BEA samples all have two peaks in the MIR intratetrahedral asymmetric stretch region, compared to four peaks seen in the As-made CIT-6, Aluminum, and Zn CIT-6 samples. The changes in the FIR from the As-made CIT-6 result in an additional intratetrahedral bending mode and a new doublering mode. The zinc ion-exchange process did not alter the As-made CIT-6 structure. Broadening of Aluminum and $\mathrm{Zn}$ CIT-6 samples in the FIR shows that chemical treatments and ion exchange disrupt the local order of the structure. The changes in the local order are difficult to see using XRD but are visible with FIR.

This work demonstrates the utility of IR, for qualitative analysis of chemical effects on zeolite structures as a complement to XRD. In particular, FIR provides information regarding how short- and long-range structures of the samples are modified by thermal or chemical treatment. Significant changes in the FIR of the samples are easier to identify than changes visible using XRD. 


\section{Acknowledgments}

The authors thank Dr. Mark Davis (California Institute of Technology) and Dr. John McKeen (formerly at California Institute of Technology) for providing the original CIT-6 sample. This project was funded by Kansas State University. Support for SRT was provided through the National Science Foundation (CBET-0943087).

\section{References}

[1] M. E. Davis, "Ordered porous materials for emerging applications," Nature, vol. 417, no. 6891, pp. 813-821, 2002.

[2] H. Kosslick and R. Fricke, "Chemical analysis of aluminosilicates, aluminophosphates and related molecular sieves," in Characterization II, H. Kosslick and R. Fricke, Eds., vol. 5 of Molecular Sieves, pp. 1-66, Springer, Berlin, Germany, 2007.

[3] G. Valerio, A. Goursot, R. Vetrivel, O. Malkina, V. Malkin, and D. R. Salahub, "Calculation of ${ }^{29} \mathrm{Si}$ and ${ }^{27} \mathrm{Al}$ MAS NMR chemical shifts in zeolite- $\beta$ using density functional theory: correlation with lattice structure," Journal of the American Chemical Society, vol. 120, no. 44, pp. 11426-11431, 1998.

[4] P. Andy and M. E. Davis, "Dehydrogenation of propane over platinum containing CIT-6," Industrial and Engineering Chemistry, vol. 43, no. 12, pp. 2922-2928, 2004.

[5] C. Baerlocher and L. B. McCusker, 2009, http://www.izastructure.org/databases.

[6] T. Takewaki, L. W. Beck, and M. E. Davis, "Zincosilicate CIT-6: a precursor to a family of * BEA-type molecular sieves," Journal of Physical Chemistry B, vol. 103, no. 14, pp. 2674-2679, 1999.

[7] R. Hajjar, Y. Millot, P. P. Man, M. Che, and S. Dzwigaj, "Two kinds of framework $\mathrm{Al}$ sites studied in BEA zeolite by Xray diffraction, Fourier transform infrared spectroscopy, NMR techniques, and V probe," Journal of Physical Chemistry C, vol. 112, no. 51, pp. 20167-20175, 2008.

[8] R. W. Stevens Jr., R. V. Siriwardane, and J. Logan, "In situ fourier transform infrared (FTIR) investigation of $\mathrm{CO}_{2}$ adsorption onto zeolite materials," Energy and Fuels, vol. 22, no. 5, pp. 30703079, 2008.

[9] A. Chowdhury, P. R. Thompson, and S. J. Milne, "TGA-FTIR study of a lead zirconate titanate gel made from a triol-based sol-gel system," Thermochimica Acta, vol. 475, no. 1-2, pp. 5964, 2008.

[10] J. Baltrusaitis, J. Schuttlefield, J. H. Jensen, and V. H. Grassian, "FTIR spectroscopy combined with quantum chemical calculations to investigate adsorbed nitrate on aluminium oxide surfaces in the presence and absence of co-adsorbed water," Physical Chemistry Chemical Physics, vol. 9, no. 36, pp. 49704980, 2007.

[11] M. May, M. Asomoza, T. Lopez, and R. Gomez, "Precursor aluminum effect in the synthesis of Sol-Gel Si-Al catalysts: FTIR and NMR characterization," Chemistry of Materials, vol. 9, no. 11, pp. 2395-2399, 1997.

[12] M. Endregard, D. G. Nicholson, M. Stöcker, and B. Beagley, "Cobalticenium ions adsorbed on large-pore aluminophosphate VPI- 5 studied by X-ray absorption, ${ }^{13} \mathrm{C}$ solid-state NMR and FTIR spectroscopy," Journal of Materials Chemistry, vol. 5, no. 3, pp. 485-491, 1995.

[13] J. A. Lercher and A. Jentys, "Infrared and raman spectroscopy for characterizing zeolites," in Studies in Surface Science and Catalysis, vol. 168, chapter 13, pp. 435-476, 2007.
[14] E. M. Flanigen, H. Khatami, and H. A. Szymanski, "Infrared structural studies of zeolite frameworks," in Molecular Sieve Zeolites-I, vol. 101 of Advances in Chemistry, pp. 201-229, 1971.

[15] E. M. Flanigen, "Zeolite chemistry and catalysis," in ACS Monograph, J. A. Rabo, Ed., vol. 171, pp. 80-117, ACS, Washington, DC, USA, 1976.

[16] G. Mestl and H. Knozinger, "Vibrational spectroscopy," in Handbook of Heterogeneous Catalysis, G. Ertl, H. Knozinger, F. Schuth, and J. Weitkamp, Eds., pp. 932-971, Wiley-VCH, Weinheim, Germany, 2nd edition, 2008.

[17] M. D. Baker, J. Godber, and G. A. Ozin, "Frequency and intensity considerations in the far-IR spectroscopy of faujasite zeolites: experiment and theory. metal cation vibrational assignments, site locations, and populations," Journal of the American Chemical Society, vol. 107, no. 11, pp. 3033-3043, 1985.

[18] J. Godber, M. D. Baker, and G. A. Ozin, "Far-IR spectroscopy of alkali-metal and alkaline-earth cations in faujasite zeolites," Journal of Physical Chemistry, vol. 93, no. 4, pp. 1409-1421, 1989.

[19] M. D. Baker, G. A. Ozin, and J. Godber, "Direct probe Fourier transform far-infrared spectroscopy of metal atoms, metal ions and metal clusters in zeolites," Catalysis reviews, vol. 27, no. 4, pp. 591-651, 1985.

[20] G. A. Ozin, M. D. Baker, J. Godber, and S. Wu, "Crystal-field effects on the far-infrared cation vibrations of transition metal (2+) ion exchanged faujasite zeolites," Journal of the American Chemical Society, vol. 107, no. 7, pp. 1995-2000, 1985.

[21] T. Takewaki, L. W. Beck, and M. E. Davis, "Synthesis of CIT-6, a zincosilicate with the *BEA topology," Topics in Catalysis, vol. 9, no. 1-2, pp. 35-42, 1999.

[22] D. P. Serrano, R. Van Grieken, M. E. Davis, J. A. Melero, A. Garcia, and G. Morales, "Mechanism of CIT-6 and VPI-8 crystallization from zincosilicate gels," Chemistry, vol. 8, no. 22, pp. 5153-5160, 2002.

[23] M. M. J. Treacy and J. M. Newsam, "Two new three-dimensional twelve-ring zeolite frameworks of which zeolite beta is a disordered intergrowth," Nature, vol. 332, no. 6161, pp. 249-251, 1988.

[24] Z. Huang, J. Su, Y. Guo, X. Su, and L. Teng, "Synthesis of wellcrystallized zeolite beta at large scale and its incorporation into polysulfone matrix for gas separation," Chemical Engineering Communications, vol. 196, no. 9, pp. 969-986, 2009.

[25] W. Zhang and P. G. Smirniotis, "Dealuminated zeolite-based composite catalysts for reforming of an industrial naphthenerich feedstock," Applied Catalysis, vol. 168, no. 1, pp. 113-130, 1998.

[26] A. Cantin, A. Corma, M. J. Diaz-Cabanas, J. L. Jorda, M. Moliner, and F. Rey, "Synthesis and characterization of the all-silica pure polymorph $\mathrm{C}$ and an enriched polymorph $\mathrm{B}$ intergrowth of Zeolite Beta," Angewandte Chemie, vol. 45, no. 47, pp. 8013-8015, 2006.

[27] S. Shetty, B. S. Kulkarni, D. G. Kanhere, A. Goursot, and S. $\mathrm{Pal}$, "A comparative study of structural, acidic and hydrophobic properties of Sn-BEA with Ti-BEA using periodic density functional theory," Journal of Physical Chemistry B, vol. 112, no. 9, pp. 2573-2579, 2008.

[28] M. A. Camblor, A. Corma, and S. Valencia, "Spontaneous nucleation and growth of pure silica zeolite- $\beta$ free of connectivity defects," Chemical Communications, no. 20, pp. 2365-2366, 1996.

[29] G. Busca, M. Vevilacqua, T. Armaroli, and M. Trombetta, "FTIR studies of internal, external and extra frame work sites of 
FER, MFI, BEA and MOR type protonic zeolite materials," Studies in Surface Science and Catalysis A, vol. 142, pp. 975-982, 2002.

[30] S. Yuvaraj and M. P. Palanichamy, "Characterization of chromium-substituted zeolite BEA," Bulletin-Chemical Society of Japan, vol. 75, pp. 155-160, 2002.

[31] C. Bisio, G. Martra, S. Coluccia, and P. Massiani, "FT-IR evidence of two distinct protonic sites in BEA zeolite: consequences on cationic exchange and on acido-basic properties in the presence of cesium," Journal of Physical Chemistry C, vol. 112, no. 28, pp. 10520-10530, 2008.

[32] J. P. Marques, I. Gener, P. Ayrault et al., "Infrared spectroscopic study of the acid properties of dealuminated BEA zeolites," Microporous and Mesoporous Materials, vol. 60, no. 1-3, pp. 251262, 2003.

[33] M. P. Fuller and P. R. Griffiths, "Infrared microsampling by diffuse reflectance fourier transform spectrometry," Applied Spectroscopy, vol. 34, no. 5, pp. 533-539, 1980.

[34] M. L. Myrick, M. N. Simcock, M. Baranowski, H. Brooke, S. L. Morgan, and J. N. McCutcheon, "The kubelka-munk diffuse reflectance formula revisited," Applied Spectroscopy Reviews, vol. 46, no. 2, pp. 140-165, 2011.

[35] J. M. Newsam, M. M. J. Treacy, W. T. Koetsier, and C. B. De Gruyter, "Structural characterization of zeolite beta," Proceedings of the Royal Society of London A, vol. 420, no. 1859, pp. 375405, 1988.

[36] G. Socrates, Infrared and Raman Characteristic Group Frequencies: Tables and Charts, John Wiley \& Sons, Chichester, UK, 3rd edition, 1994.

[37] S. Kinugasa, K. Tanabe, and T. Tamura, 2010, http://sdbs.riodb .aist.go.jp/sdbs/cgi-bin/direct_frame_top.cgi. 

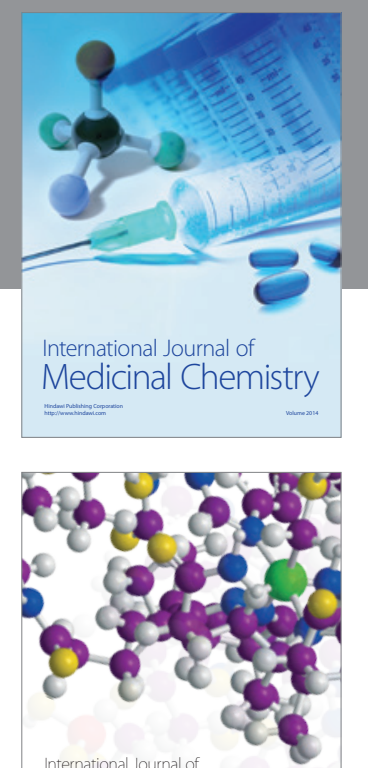

\section{Carbohydrate} Chemistry

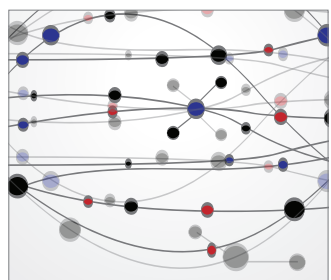

The Scientific World Journal
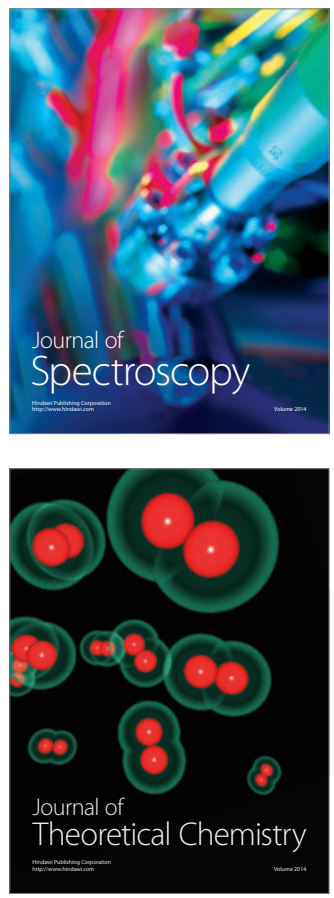
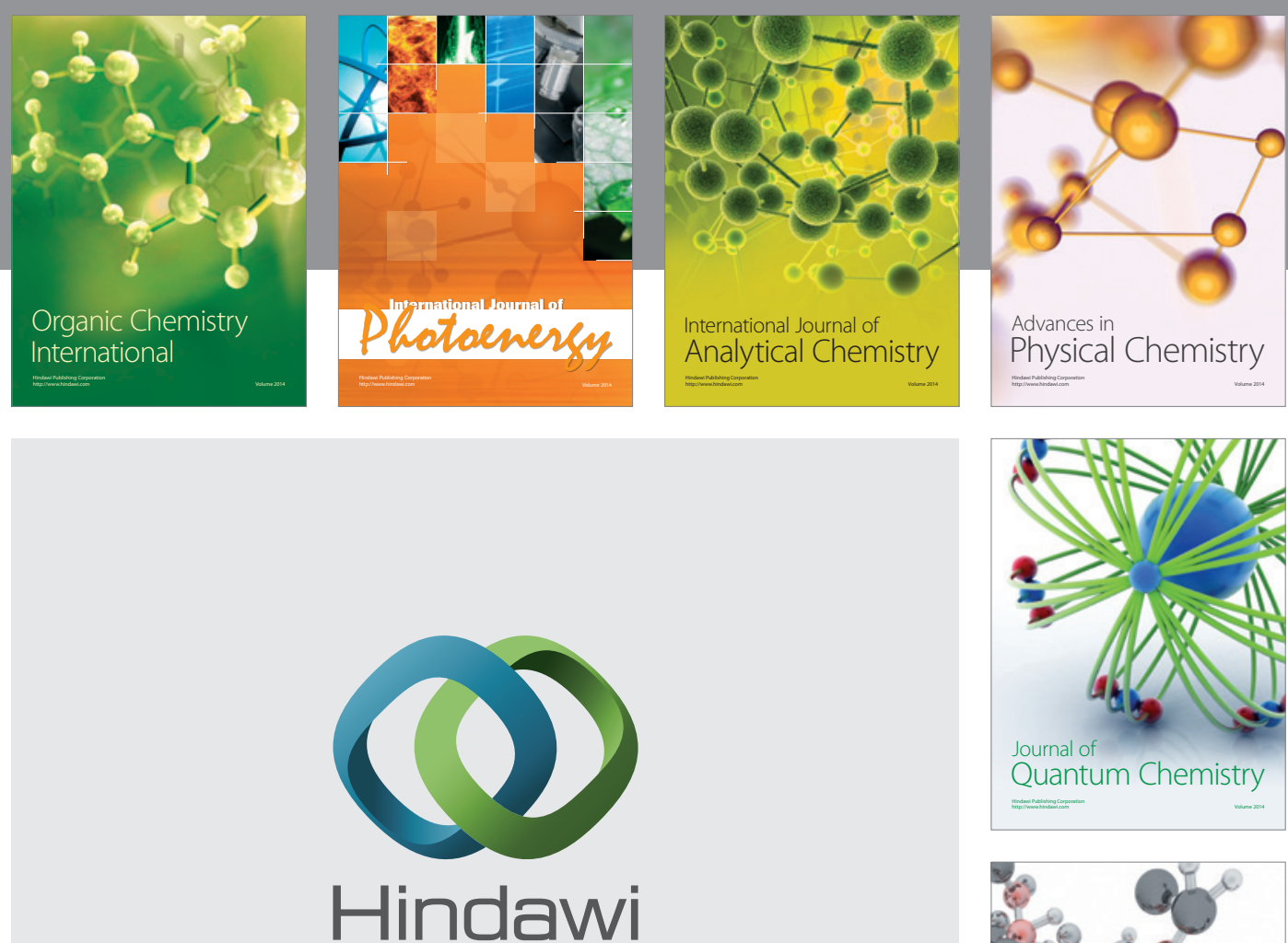

Submit your manuscripts at

http://www.hindawi.com

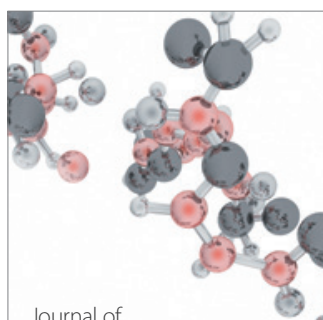

Analytical Methods

in Chemistry

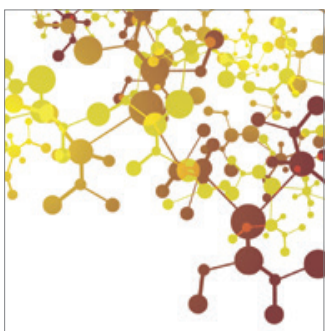

Journal of

Applied Chemistry

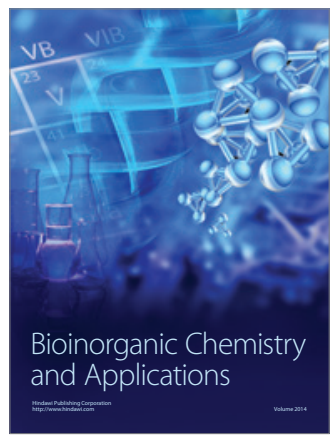

Inorganic Chemistry
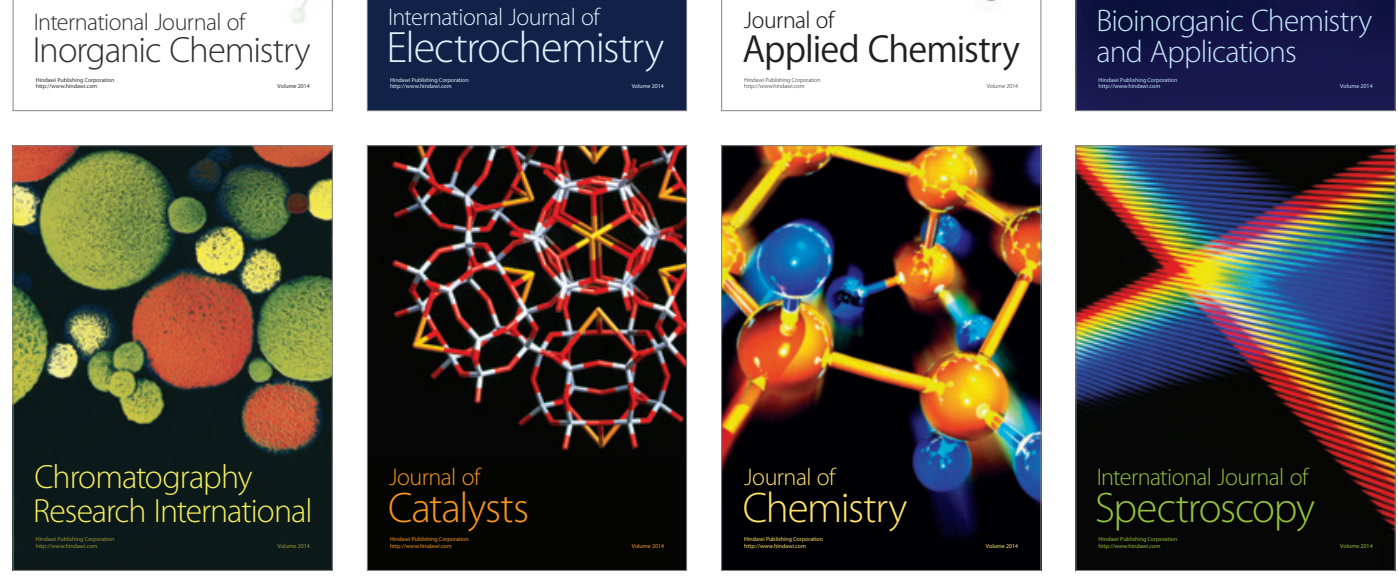